\title{
Maternal Mortality among Women Seeking Health Care Services in Kisii Level 5 Hospital
}

\author{
Osoro $\mathrm{AA}^{1, *}$, Ng'ang'a $\mathrm{Z}^{2}$, Mutugi $\mathrm{M}^{3}$, Wanzala $\mathrm{P}^{4}$ \\ ${ }^{1}$ Kisii Medical Training College, P.O. Box 1165-40200, Kisii \\ ${ }^{2}$ Jomo Kenyatta University of Agriculture and Technology, Institute of Tropical Medicine and Infectious Diseases \\ ${ }^{3}$ Jomo Kenyatta University of Agriculture and Technology, Department of Zoology \\ ${ }^{4}$ Kenya Medical Research Foundation (KEMRI), Nairobi \\ *Corresponding author: alfredosoro2013@gmail.com
}

Received August 11, 2014; Revised September 05, 2014; Accepted September 16, 2014

\begin{abstract}
Background. Maternal mortality has continued to escalate in Kenya inspite of all the efforts being made by the government, non-governmental organization and the community. The purpose of the study was to establish the antecedent factors predisposing women to maternal death using the three delays model. Methodology: Retrospective 72 maternal death cases which occurred between January 01, 2009 and June 30, 2010 were reviewed by an independent gynecologist/obstetrician. Interviews were conducted with kins of the deceased women using a confidential questionnaire for female death based on distal as well as proximal factors that may have had a bearing on maternal death. Health care workers were likewise interviewed using a formulated questionnaire to assess the ability and readiness of the hospital to offer emergency obstetric care. Results: A record of 72 maternal deaths occurred during the study period thus between January 1, 2009 and June 30. 2010. Out of 72, 42 maternal deaths were as a result of direct obstetric complications which include hemorrhage, post-partum sepsis, pre-eclampsia and abortion. Post partum hemorrhage was the most common complication which contributed to maternal deaths. Again out of 72 maternal deaths, 33 were as a result of indirect causes with peritonitis, heart disease, HIV/AIDS, anemia, and convulsive disorder respectively. Delayed access to transport, lack of money for user fees, and hospital distance were challenges that led to delay in accessing care. Hospital experiences included; delay in service provision by staff, delayed quality emergency obstetric care and delayed care while at the hospital, unavailability of blood for transfusion, and lack of money for drugs, were reported as major challenges which attributed to maternal death. Conclusion: The study suggests maternal mortality occurs as a result of lack of access to quality healthcare facility poor health seeking behavior and poor socio-economic factors.
\end{abstract}

Keywords: maternal mortality, confidential female death questionnaire, verbal autopsy

Cite This Article: Osoro AA, Ng'ang’a Z, Mutugi M, and Wanzala P, "Maternal Mortality among Women Seeking Health Care Services in Kisii Level 5 Hospital.” American Journal of Public Health Research vol. 2, no. 5 (2014): 182-187. doi: 10.12691/ajphr-2-5-1.

\section{Introduction}

Globally, nearly eight million women suffer from complications of pregnancy and childbirth and approximately 600,000 women die while giving birth annually [1]. According to the World Health Organization, while over 350 women per 100000 live births die of avoidable maternal deaths, more than 8 million women suffer complications related to pregnancy and childbirth every year in the developing world [1]. However, maternal mortality ratio has decreased from 1990 to 2010 (1100 to 675 per 100,000 live births, respectively) worldwide [2]. In 2010, maternal mortality rate (MMR) had decreased to 210 maternal deaths per 100,000 live births, down from 400 maternal deaths per 100,000 live births in 1990 globally [2,5]. In Sub-Saharan Africa, the lifetime risk of maternal death for women is 1 in 39, in the United States, it is 1 in 2,400 while in Sweden it is 1 in 14,100. In Kenya, the incidence of maternal mortality rate is 488 per 100,000 live births [4], while in Kisii it is estimated to be 500 per 100,000 live births [6]. This is indeed above the national average. It is unlikely that Kenya will reach the Millennium Development Goal (MDGs) 5 target of 147 maternal deaths per 100,000 live births by 2015 [5]. In Kisii it is estimated to be 500 deaths per 100,000 live births [6], which is above the national average.This underscores the need for urgent and targeted investments in the hardest-hit countries and particularly in Kenya.

The efforts that have been made by member states and international organizations to reduce maternal mortality rates and improve the health of women and children to achieve the Millennium Development Goal number four and five by 2015 have not had significant improvement especially in developing countries [7]. The numbers of women who die or suffer from morbidity following childbirth complications reveal that maternal health is extremely poor in many countries of the world. A tool that can be used to explore factors contributing to maternal 
mortality is "Confidential Female Death Inquiries". Information received can help policy-makers to formulate policy guidelines and interventions at individual, community, facility, and political level. The tool can reveal the characteristics of the women, cause of death, associated risk factors and delays to case [6]. Delays in decision making, reaching an appropriate obstetric facility and receiving quality obstetric care while in the facility have been studied [7].

Understanding maternal mortality through identification of associated risk factors and verbal autopsy has been shown to contribute to policies and interventions that reduce maternal mortality [8]. Maternal mortality is a major public health concern and is an indicator of the standard of health care system of many countries $[9,10]$. The purpose of the study was to identify the predictors of maternal mortality from previous pregnancies which have contributed to maternal death in Kisii County.

\section{Material and Methods}

\subsection{Study Design}

A descriptive retrospective study design was conducted collecting both qualitative and quantitative data.

Study setting: Kisii General Hospital, a Level 5 Referral Hospital.

Kisii General Hospital is a "Level 5" referral facility of the neighbouring sub-county hospitals which include Rachwonyo Sub-county of Homa-Bay County, Nyamira County, Gucha sub-county, Masaba sub-county, South Kisii sub-county, Transmara sub-county, Homa-Bay County and Migori sub-county. The General Hospital has fairly adequate facilities with at least one consultant in the core branches of medicine, surgery, pediatricsand obstetrics and gynaecology. It has fairly adequate nursing staff that is capable of providing services to the patients. It has among others; a maternity theatre and other three theatres for general surgeries. Referral facilities exist and the needy cases are referred to the Kenyatta National Hospital in Nairobi or Moi Teaching and Referral Hospital in Eldoret. It has a daily outpatient attendance of 400 people with a bed capacity of 500 for inpatients. The hospital records an average of 12 deliveries and 3 caesarean operations per day (Hospital Records, 2009).

\subsection{Study Population}

The study populations comprised of relatives of women who had died as a result of pregnancy and childbirthrelated complications while seeking for healthcare services at Kisii Level 5 Hospital from January 01, 2009 to June 30, 2010.

\subsection{Ethical Consideration}

Ethical approval was granted by the Ethical Research Committee from Kenya Medical Research Institute (KEMRI) Protocol No.1851 of November 2010. Permission to conduct the study was obtained from the Provincial Director of Medical Services Nyanza and copied to the medical officer of Health- Kisii Level 5 Hospital. The area Chief was informed. Those who participated in the study were requested to give informed voluntary consent and were assured of confidentiality of information obtained from them. Adequate arrangements were made available for counseling services in anticipation for any psychological or emotional issues arising, as a result of the nature of the study. A structured questionnaire was administered to the immediate adult family member, who was present and attended to the deceased, thus the husband and if he was unavailable, then the mother-in-law or a close relative. The interview was conducted by a registered nurse with two clinical officers in an identified room with no movement of people. The research assistants introduced themselves to the family of the deceased woman, expressing condolences for the death of a beloved one. The purpose of the visit was then stated as to try and establish the circumstances which led to her death. Information of her condition prior to her death was sought with the explanation that this was important, to prevent others like her from dying in similar circumstances. This would thus stop further tragic happenings.

\subsection{Data Variables}

Socio-demographic variable included: Categorized age: 15-20, 21-25, 26-30, 31-35, and 36-45. Marital status: married, single, separated, widowed. Educational Levels: none, primary, secondary, college/tertiary. Religious faith: catholic, protestant and others-SDA. Occupation: none, farmer, housewife, businesswoman, formal employment. Residence: rural, urban and periurban.

Underlying factors to maternal mortality (Complications): Spotting of blood, swelling of hands and legs, bleeding, hypertension, heart disease, convulsions, infections/sepsis, HIV/AIDS.

Risk factors antecedent to maternal mortality: Previous pregnancies planned, duration of previous pregnancies, abortion; State of pregnancy: Term, preterm, abortion, doubtful. Nature of delivery: Normal, breech, surgery. Person who conducted delivery: Doctor, nurse/midwife, traditional birth attendant, and self.

Place of birth: Hospital (Public healthcare institution hospital, health centre, dispensary), private health facility or home.

Outcomes of pregnancy and childbirth complications antecedent to maternal death: Not seeking healthcare services promptly when labor started, lack of transport in spite of availability of money, lack of money for user fees, long distance to the healthcare facility more than 12 kilometers, no specific reason for not seeking prompt healthcare services.

\subsection{Data Collection Methods and Tools}

All files of maternal deaths which occurred during the study period were sorted out and recorded in an entry proforma in the order in which they occurred. These included the age, parity, socio-economic and sociodemographic characteristics, booking status, referral sources and cause of death. The three delays were equally documented. A Confidential Questionnaire of Household (CQH) (Q3) - based on verbal autopsy technique, was used to interview and collect information from relatives or significant others associated with the deceased women. 
A structured questionnaire was administered to the healthcare workers to establish the ability of the institution in providing emergency obstetric care, the state of preparedness of the staff to cope up with the workload in emergency situations, and the availability of referral facility.

Data cleaning was done using a standardized procedure, whereby extreme values and impossible answers were deleted. Specifically, data was explored using descriptive statistics (histogram, stem and leaf, cross-tabs) to check for inconsistencies. Finally, coding was verified to only correspond to what is possible. After data cleaning, analysis was done using computer software "Statistical Package for Social Scientist (SPSS)” version 16.0. Descriptive analysis was done in which percentage was used to describe factors associated with maternal mortality; results were presented in a table and bar graphs.

\section{Results}

\subsection{Socio-Demographics}

During the study period (January 01, 2009 and June 30, 2010), 72 cases of maternal deaths occurred, ranging from 15 to 45 years of age. Majority, 29 (40.3\%) were between 21 to 25 years of age. Majority of the deceased 47 (65.3\%) were married, while 21 (29.2\%) were single. As regards to their occupation, housewives formed the bulky 29 (40.3\%) while 19 (26.4\%) were farmer and 18 (25\%) were businesswomen.

Majority of these deceased women 50 (69.4\%) were from the rural areas, while 15 (20.8\%) were from the urban area and 7 (9.7\%) were from the peri-urban area, See Table 1 which show socio-demographic characteristics of the deceased women.
Table 1. Socio-demographic characteristics $(\mathrm{N}=72)$

\begin{tabular}{llll}
\hline Characteristics & & $\mathrm{N}$ & $\%$ \\
& $15-20$ & 14 & 9.4 \\
Age in Years & $21-25$ & 29 & 40.3 \\
& $26-30$ & 21 & 29.2 \\
& $31-35$ & 7 & 9.7 \\
& $36-45$ & 1 & 1.4 \\
Marital Status & Single & 21 & 29.2 \\
& Married & 47 & 65.3 \\
& Separated & 3 & 4.2 \\
Education & Widowed & 1 & 1.4 \\
& None & 5 & 6.9 \\
Religion & Primary & 5 & 6.9 \\
& Secondary & 33 & 45.8 \\
& College/tertiary & 29 & 40.3 \\
& Catholic & 35 & 48.6 \\
Residence & Protestant & 33 & 45.8 \\
& Others (SDA) & 4 & 5.6 \\
Occupation & None & 2 & 2.8 \\
& Farmer & 19 & 26.4 \\
& Housewife & 29 & 40.3 \\
& Businesswoman & 18 & 25.0 \\
& Formal employment & 4 & 5.6 \\
& Rural & 50 & 69.4 \\
& Urban & 15 & 20.8 \\
& Peri-urban & 7 & 9.7 \\
\hline
\end{tabular}

\subsection{Underlying Factors to Maternal Mortality}

More than a third of the subjects (80\%) experienced spotting/bleeding, 73.5\% experienced swelling of hands and feet, $64.4 \%$ had severe bleeding in pregnancy, while $21.6 \%$ had raised blood pressure. The study also revealed that $43.5 \%$ of the subjects had pre-existing heart disease, $41.5 \%$ had convulsive disorders while $43.1 \%$ had postpartum infections. See Figure 1, underlying factors to maternal mortality.

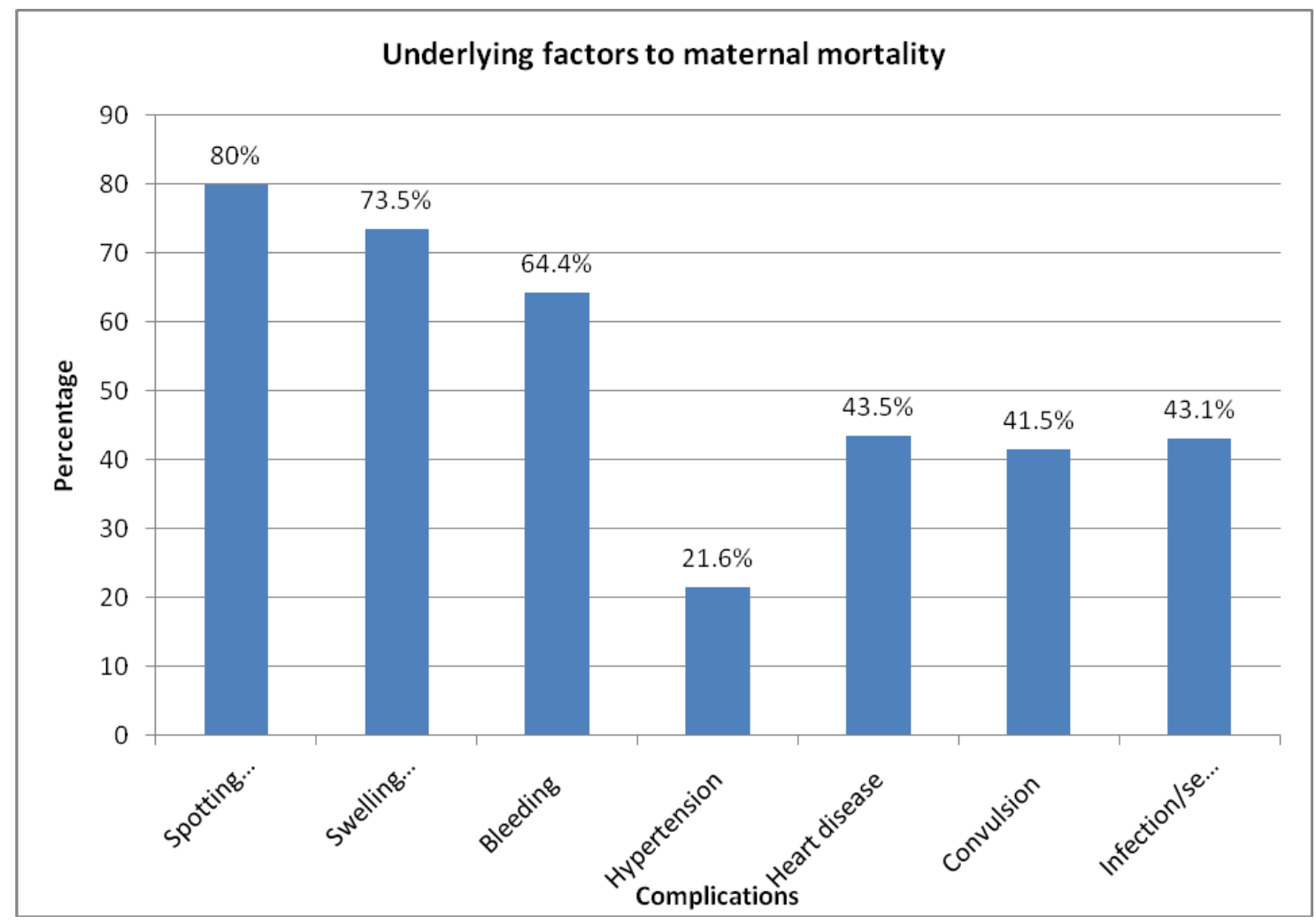

Figure 1. Underlying factors to maternal mortality as described by relatives of deceased women or from verbal autopsy 


\subsection{Immediate Risk Factors Associated with Maternal Mortality}

Out of 72 subjects, 38 didn't plan for their pregnancies, again, 20 pregnancies ended in abortion.

Fourteen deliveries were preterm, while 25 deliveries occurred at home and 51 mothers didn't go promptly to hospital when labor started. Sixty-one had normal delivery, 8 of them were operated, while 3 were breech deliveries. Although majority of the deliveries were normal, they were accompanied with risk factors that led to maternal death. See Table 2. This shows the immediate risk factors associated with maternal mortality.

Table 2. Shows immediate factors associated with maternal mortality $(\mathrm{N}=72)$

\begin{tabular}{|l|l|l|l|}
\hline & & N & \% \\
\hline $\begin{array}{l}\text { Previous pregnancies } \\
\text { planned }\end{array}$ & No & 38 & 52.8 \\
& Yes & 32 & 44.4 \\
& Doubtful & 2 & 2.8 \\
\hline \multirow{2}{*}{ Aborted } & No & 52 & 72.2 \\
Duration of previous & Yes & 20 & 27.8 \\
\hline pregnancies & Term & 14 & 19.4 \\
& Preterm & 44 & 61.2 \\
Location of previous & Dispensary & 14 & 19.4 \\
\hline deliveries & Health centre & 25 & 34.7 \\
& Hospital & 7 & 9.7 \\
\hline $\begin{array}{l}\text { Go promptly to } \\
\text { hospital when labour } \\
\text { started }\end{array}$ & No & 23 & 23.6 \\
\hline \multirow{2}{*}{$\begin{array}{l}\text { Type of delivery or } \\
\text { birth }\end{array}$} & Yes & 51 & 70.8 \\
\hline
\end{tabular}

Foot note:

* Breech delivery- the position of a baby in the uterus such that it will be delivered buttocks first (instead of the normal head-first position). This type of delivery increases the risk of damage to the baby. Normal delivery-the position of the baby in the uterus such that it will be delivered head first (this is the normal position which many babies adopt during delivery).

\subsection{Outcome of Pregnancy and Childbirth Complications Antecedent to Maternal Death}

The indirect causes of maternal mortality as reported by the adult kins of the deceased and collaborated by the obstetrician were; pre-eclampsia, bleeding, postpartum sepsis, pregnancy-induced hypertension and heart disease. Persons who conducted the deliveries included: doctor (16.7\%), nurse/midwife (44.4\%), traditional birth attendants (23.6\%), relative (4.2\%), and woman delivered herself (11.1\%). From these findings, many deliveries were conducted by skilled attendants and other key players yet they had risk factors which led to maternal death.

\subsection{Risk Factors and Complications Antecedent to Maternal Mortality}

Delayed hospitalization, unplanned pregnancies, and obstetric complications, abortion, not being seen by the doctor, surgery and preterm deliveries were risk factors antecedent to maternal mortality. Most of the subjects 51
(70.8\%) did not seek prompt healthcare services as reported by relatives.

The study confirmed from relatives that lack of means of transport, lack of money for user fees and the long distances to the hospital were the main reason for not seeking healthcare promptly. However, 17 (23.6\%) subjects had no reason as to why they did not seek prompt healthcare.

\subsection{Factors that Affected the Accessibility of Maternal Health Services}

Delay in accessing healthcare services from the healthcare providers was reported by 14 (19.4) subjects while $6(8.3 \%)$ subjects reported lack of blood for transfusion following severe bleeding and 12 (16.7\%) subjects had no money for purchasing prescription drugs.

All the deceased women were subjected to the "Three Delays" process which explained the circumstances that took place prior to death. Some of the deceased women ended up to "Four Delays" as a result of the healthcare workers miss-managing them from subsequent delays particularly managing the complications.

\section{Discussions}

We retrospectively investigated antecedent factors which contributed to maternal mortality among women of reproductive age seeking healthcare services at Kisii Level 5 Hospital.

\subsection{Demographic Characteristics}

Majority of women who succumbed to maternal mortality were young females (21-25 years). This is a young vulnerable group in the prime of their life and this is indeed tragic to society. In a study by WHO, it reported that adolescent girls between the age of 15-24 are vulnerable to maternal death because of lack of antenatal care [15]. Mortality among this group and their children is a global collective failure [16], and dying in pregnancy or childbirth is common among teenage girls in developing countries [6].

\subsection{Causes of Maternal Mortality}

Bleeding, heart diseases, infection/sepsis and convulsive disorders were the major findings revealed by the study. These findings concur with other previous studies by Petro-Barvazian [17], except that heart diseases did not feature in them. This is probably a unique local variation in the study area that may require additional studies. One other salient finding in this study was abortion cases (27.8\%) for which a distinctive pattern emerged among women aged 15 to 19 years. This is age groups who are at school and have no access to contraceptives. They would resort to abortion so that they can continue with their education only to end up as mortality statistics. Indeed the death rate from this group may be more than it is represented here since it is considered an illegal activity [15].

Majority of the subjects were attended by skilled birth attendants yet they succumbed to death, while other deliveries were conducted at home where the women were 
exposed to risk factors which may have led them to death. This shows that maternal deaths take place in both hospitals and rural homes. Mothers seek healthcare services when they are in bad obstetric or health condition with co-morbidities which led them to maternal death. Some of the delays to seek health care services promptly can be as a result of late referral from lower health facilities and delayed in decision-making, either from the healthcare worker or relatives or both. Various beliefs and customs affect the use of health facilities, for example, in Kisii community; delivery has to be conducted by elderly mothers. Most of the women deliver at home under unhygienic conditions, exposing them to greater risk of death.

Out of the seventy-two deceased women, 23.6\% had no reported reason as to why they did not seek obstetric care. This can be attributed to health seeking behavior. The inaction contributed to delay in delayed interventions [7]. It has been observed that women do not seek healthcare services due to probably lack of independence in decisionmaking process on key issues affecting their own lives, for example, decision-making process is influenced by the husband, mother-in-law and occasionally by family members regarding where a woman/mother can deliver. Similarly, this study agrees with a study done in Pakistan by Ashford [22] where a third of women who delivers at home do so because their husband or other family members forbade hospital delivery, while many women in southern Asia and parts of Africa need permission to visit a health facility or must be escorted when husband is away from home. Women rarely use even nearby health services. These women when they develop complications, by the time they reach the hospital they have risk factors which led them to death.

Lack of means of transport, lack of money, and hospital being far, were some of the challenges that negated accessing care. Transport can be a nightmare especially at night when labor start and the health facility is far and none in the neighborhood has a vehicle even if money is available. Those trying to reach a far-off facility, fail and women with serious complications may die en route. This is made worse when combined with lack of transport and poor impassible roads especially during rainy seasons.

Limited access to financial resources is a major limiting factor among women and this impact negatively on control over their lives. In situations where women have no control over their own or their family income, their ability to use maternity services especially where user fees are involved are further constrained [19]. Therefore, the economic dependence of women on their husbands has direct influence on their lives for it would hinder the use of maternity care service facilities even when available. However, this is not only endangering their lives but also the lives of the newborn babies as well [19]. The study showed that majority of women (69.4\%) live in rural areas which have limited skilled attendants; they seek the services of the TBAs thus, citing the reason for their delay. This concurs with another study [20] which showed that some of the skilled attendants are few and some have bad attitude that although they are trained, many women feel that they have insufficient and inadequate skills.

Unplanned pregnancies were revealed by the study as a challenge that can be addressed by access to contraceptives. The study revealed that $27.8 \%$ had abortion and this correlated with another study [21], which report that $13 \%$ of all maternal deaths occur among adolescents mainly as a result of complications of unsafe abortion.

Delay in service provision by staff was cited as a challenge. They reported that few staff were on duty quite busy with other patients, while they waited for their turn. Lack of blood for transfusion and lack of money for prescription drugs negated provision of quality services. These are institutional problems that are common, and negate emergency obstetric care. However, many institutions are not functional due to frequent stock-outs, lack of equipment, essential supplies and qualified staff [7]. This information was collaborated and confirmed by the healthcare worker's questionnaire. This may have necessitated the choice of non-orthodox delivery services by these pregnant women.

\section{Conclusions}

The study suggests that the increase in maternal mortality is due to poor access of women to quality healthcare services. Improving socio-economic status of women and empowerment in decision-making, antenatal care, increasing access to skilled delivery, increasing contraceptive uptake and strengthening healthcare system, will scale down the burden of maternal mortality.

However, this study need to be followed by other large scale community- based study, which can provide useful data to inform the healthcare authorities, and communityleaders to plan appropriate interventions to reduce maternal mortality. The limitation of this study is that no control group has been studied, which could have helped in statistically comparing the socio-demographic characteristics, the antecedent factors and the frequency of delays between mothers who died and those who lived.

\section{Acknowledgement}

This work received partial support from "Strengthening Nurses' Capacity in HIV Policy Development in SubSaharan Africa and the Caribbean funded by the Global Health Research Initiative (GHRI), a collaborative research funding partnership of the Canadian Institutes of Health Research, the Canadian International Development Agency, Health Canada, the International Development Research Centre, and the Public Health Agency of Canada.

\section{Conflict of Interest}

The authors have no conflicts of interest.

\section{References}

[1] WHO (2004). Beyond the Numbers: Reviewing Maternal Death and Complications to Make Pregnancy Safer, WHOPress, Geneva.

[2] WHO; UNICEF and the World Bank (2012). Trends in maternal mortality 1990-2010, Geneva, WHO.

[3] UNFPA, WHO, UNICEF and the World Bank. (2011). Trends in maternal mortality: 1990 to 2010. Released new report. 
[4] Kenya National Bureau of Statistics (KNBS) and ICF Macro. (2010). Kenya Demographic and Health Survey 2008-09. Calverton, Maryland: KNBS and ICF Macro.

[5] Kenya Demographic and Health Survey (2008/9). Calverton, Maryland, CBS, MoH and ORC Macro, 2004

[6] Yumiko K. (2008). A Manual for partnership workshops. Samokike project: JapaneseInternational Co-operation Assistance - Kisii /Kericho Project.

[7] Thaddeus S, Maine D. (1994).Too far to walk: maternal mortality in context. Social Science Medicine 38: 1091-1110.

[8] van Dillon, J.S., Stekelenburg, J., Schutte, J., Walraven, G., van Roosmalen, J. (2007). The use of audit to identify maternal mortality in different settings: is it just a difference between the rich and the poor? Healthcare Quarterly 10, 131-137.

[9] United Nations; WHO. (2000). World Bank Partner to combat Maternal Mortality the World Bank Group News Release No. 2000/080/S

[10] WHO; United Nations Millennium Project, UNDP (2005). WHO (2005). Defining Maternal Death: Africa Journal of Medical Practice, (2005) 12 (2) pp 33. Vol. 12 No. 2 April 2005 Accelerating Progress and Expanding efforts to improve Maternal Health. UN/UNDP 2005 Progress Report.

[11] WHO (1996). Prenatal Mortality. Document FRH/MSM/96.7 Geneva: WHO, 1996

[12] Penney G.and Brace V., (2007). Near miss audit in obstetrics. Current Opinion in Obstetrics and Gynecology 19, 145-150.
[13] United Nations (2009). Accelerating progress and expanding efforts to improve Maternal Health. UN/UNDP Progress Report.

[14] Chandramohan D., Gillan H., Rodrigues L. (2001). Verbal autopsy for adults deaths: development and validation in a multicentre study. Tropical Medicine and International Health.8; 437-46.

[15] UNFPA and UNICEF (2000). Situational Analysis of women and children in Kenya.

[16] W.J. Graham (2010). Maternal Mortality: Evidence Gap and Measurement Traps. Journal of Obstetrics and Gynaecology.

[17] Petros-Barvazian A. (1984). World Priorities and Targets in Maternal and Child Health for the Year 2000, International Journal of Gynaecology \& Obstetrics. IJGOA 22 (6) 429-505.

[18] Fillippi V, Ronsmans C, Gohou V, et al. (2005). Maternity wards or emergency Obstetric rooms? Incidence of near-miss events in African hospitals. Acta obstetrician et Gynecologica Scandinavica 84, 11-16.

[19] WHO (1999). Maternal Mortality and Morbidity Report, World Health Organization. Geneva, Switzerland.

[20] WHO (2001). WHO Bulletin: Managing Maternal Mortality: Special series. Geneva, Switzerland.

[21] WHO (2008). "Why is giving special attention to adolescents important for achieving Millennium Development Goal 5?” Adolescent Pregnancy fact sheet. Geneva: department of making pregnancy safe, WHO.

[22] Ashford L. (2001). Resource Flows for International Population Assistance and UNFPA. 